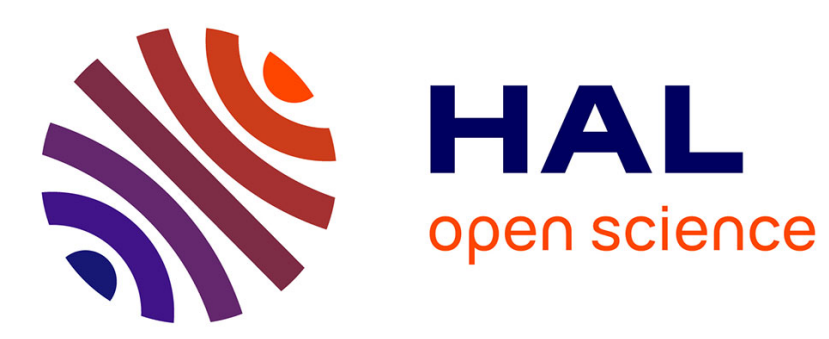

\title{
Direct derivation of the stochastic CRB of DOA estimation for rectilinear sources
}

Habti Abeida, Jean-Pierre Delmas

\section{To cite this version:}

Habti Abeida, Jean-Pierre Delmas. Direct derivation of the stochastic CRB of DOA estimation for rectilinear sources. IEEE Signal Processing Letters, 2017, 24 (10), pp.1522 - 1526. 10.1109/LSP.2017.2744673 . hal-01629444

\section{HAL Id: hal-01629444 \\ https://hal.science/hal-01629444}

Submitted on 6 Nov 2017

HAL is a multi-disciplinary open access archive for the deposit and dissemination of scientific research documents, whether they are published or not. The documents may come from teaching and research institutions in France or abroad, or from public or private research centers.
L'archive ouverte pluridisciplinaire $\mathbf{H A L}$, est destinée au dépôt et à la diffusion de documents scientifiques de niveau recherche, publiés ou non, émanant des établissements d'enseignement et de recherche français ou étrangers, des laboratoires publics ou privés. 


\title{
Direct derivation of the stochastic CRB of DOA estimation for rectilinear sources
}

\author{
Habti Abeida and Jean Pierre Delmas, Senior member, IEEE
}

\begin{abstract}
Several direction of arrival (DOA) estimation algorithms have been proposed to exploit the structure of rectilinear or strictly second-order noncircular signals. But until now, only the compact closed-form expressions of the corresponding deterministic Cramér Rao bound (DCRB) have been derived because it is much easier to derive than the stochastic CRB (SCRB). As this latter bound is asymptotically achievable by the maximum likelihood (ML) estimator, while the DCRB is unattainable, it is important to have a compact closed-form expression for this SCRB to assess the performance of DOA estimation algorithms for rectilinear signals. The aim of this paper is to derive this expression directly from the Slepian-Bangs formula including in particular the case of prior knowledge of uncorrelated or coherent sources. Some properties of these SCRBs are proved and numerical illustrations are given.
\end{abstract}

Index Terms-Deterministic and stochastic Cramér Rao bound (CRB), direction of arrival (DOA), circular, noncircular, rectilinear, strictly noncircular, Slepian-Bangs formula.

\section{INTRODUCTION}

Various DOA estimation algorithms such as MUSIC [1], [2], root-MUSIC [3], standard ESPRIT [4] and unitary ESPRIT [5], [6] have been adapted to exploit the structure of rectilinearity or strictly second-order noncircularity of signals, which include commonly used digital modulation schemes such as BPSK and ASK. These algorithms are known to achieve a higher estimation accuracy and can resolve up to twice as many sources compared to the traditional DOA algorithms. To assess the performance of these algorithms, it is necessary to derive the SCRB for rectilinear sources. Nonetheless, only the SCRB for arbitrary noncircular sources [7], [8] and the DCRB for rectilinear sources [9]-[11] are available, among many other bounds (e.g., [12] and references therein). But the first bound does not take into account the prior knowledge of rectilinearity and the second bound, although providing valuable engineering insight is unattainable.

As generally the exploitation of prior knowledge usually reduces the estimation error, this paper derives closed-form expressions of the SCRB for arbitrary rectilinear sources and for the specific prior knowledge of uncorrelated, and

Habti Abeida is with Dept. of Electrical Engineering, University of Taif, Al-Haweiah, 21974, Saudi Arabia, e-mail: abeida3@yahoo.fr

Jean-Pierre Delmas is with Telecom SudParis, UMR CNRS 5157, Université de Paris Saclay, 91011 Evry Cedex, France, e-mail: jeanpierre.delmas@it-sudparis.eu, phone: +(33).1.60.76.46.32. fully correlated (referred to coherent) sources. Note that explicit expressions of circular and noncircular SCRBs for DOA parameter alone have been derived by two different methods for arbitrary sources. The first one consists of computing the asymptotic covariance matrix of the concentrated ML estimator [13], which is asymptotically efficient and the other one is obtained directly from the SlepianBangs formula [14], [15]. Similarly to [16], we present here a direct derivation of the different rectilinear SCRBs from the extended Slepian-Bangs formula [7] for noncircular Gaussian distributions. Finally some properties of these SCRBs are proved and numerical illustrations are given.

\section{DATA MODEL AND PROBLEM FORMULATION}

Consider $K$ zero-mean narrowband signals $\left(x_{t, k}\right)_{k=1, \ldots, K}$ impinging on an arbitrary array of $M$ sensors. These signals are supposed rectilinear (also called strictly second-order noncircular), i.e., described by the following model:

$$
x_{t, k}=s_{t, k} e^{i \phi_{k}} \text { with } s_{t, k} \text { real-valued, }
$$

where the phases $\phi_{k}$ associated with different propagation delays are assumed fixed, but unknown during the array observation. The array output at time $t$ is modeled as

$$
\mathbf{y}_{t}=\mathbf{A}_{\theta} \boldsymbol{\Delta}_{\phi} \mathbf{s}_{t}+\mathbf{n}_{t}, \quad t=1, \ldots, T,
$$

where $\left(\mathbf{y}_{t}\right)_{t=1, \ldots, T} \quad$ are independent. $\quad \mathbf{A}_{\theta} \quad \stackrel{\text { def }}{=}$ $\left[\mathbf{a}\left(\theta_{1}\right), \ldots, \mathbf{a}\left(\theta_{K}\right)\right]$ denotes the conventional steering matrix, $\boldsymbol{\Delta}_{\phi} \stackrel{\text { def }}{=} \operatorname{Diag}\left(e^{i \phi_{1}}, \ldots, e^{i \phi_{K}}\right)$ and $\mathbf{s}_{t} \stackrel{\text { def }}{=}\left(s_{t, 1}, \ldots, s_{t, K}\right)^{T}$. $\mathbf{n}_{t}$ is the additive noise, which is assumed zero-mean circular complex Gaussian, spatially uncorrelated with $\mathrm{E}\left(\mathbf{n}_{t} \mathbf{n}_{t}^{H}\right)=\sigma_{n}^{2} \mathbf{I}$ and independent from $s_{t, k}$. $\left(s_{t, k}\right)_{k=1, \ldots, K, t=1, . . T}$ are either real-valued deterministic unknown parameters (in the so-called conditional or deterministic model), or zero-mean real-valued Gaussian distributed with covariance $\mathrm{E}\left(\mathbf{s}_{t} \mathbf{s}_{t}^{T}\right)=\mathbf{R}_{s}$ (in the so-called unconditional or stochastic model).

To derive the CRB from the Slepian-Bangs formula, we have to carefully specify the parameters of the Gaussian distribution of $\left(\mathbf{y}_{t}\right)_{t=1, \ldots, T}$. Under the deterministic assumption, $\mathbf{y}_{t}$ are circularly Gaussian distributed with mean $\left(\mathbf{A}_{\theta} \boldsymbol{\Delta}_{\phi} \mathbf{s}_{t}\right)_{t=1, \ldots, T}$ and covariance $\sigma_{n}^{2} \mathbf{I}$, which are parameterized by the real-valued parameter:

$$
\boldsymbol{\alpha}=\left(\boldsymbol{\theta}^{T}, \boldsymbol{\phi}^{T}, \boldsymbol{\rho}^{T}, \sigma_{n}^{2}\right)^{T},
$$


where $\boldsymbol{\theta} \stackrel{\text { def }}{=}\left(\theta_{1}, \ldots, \theta_{K}\right)^{T}, \boldsymbol{\phi} \stackrel{\text { def }}{=}\left(\phi_{1}, \ldots, \phi_{K}\right)^{T}$ and $\boldsymbol{\rho} \stackrel{\text { def }}{=}$ $\left(\mathbf{s}_{1}^{T}, \ldots, \mathbf{s}_{T}^{T}\right)^{T}$. On the other hand, under the stochastic assumption, $\mathbf{y}_{t}$ is noncircularly Gaussian distributed with zero-mean, covariance

$$
\mathbf{R}_{y} \stackrel{\text { def }}{=} \mathrm{E}\left(\mathbf{y}_{t} \mathbf{y}_{t}^{H}\right)=\mathbf{A}_{\theta} \boldsymbol{\Delta}_{\phi} \mathbf{R}_{s} \boldsymbol{\Delta}_{\phi}^{*} \mathbf{A}_{\theta}^{H}+\sigma_{n}^{2} \mathbf{I}
$$

and complementary covariance

$$
\mathbf{C}_{y} \stackrel{\text { def }}{=} \mathrm{E}\left(\mathbf{y}_{t} \mathbf{y}_{t}^{T}\right)=\mathbf{A}_{\theta} \boldsymbol{\Delta}_{\phi} \mathbf{R}_{s} \boldsymbol{\Delta}_{\phi} \mathbf{A}_{\theta}^{T},
$$

which is also generally parameterized by (3), but where $\rho$ is now the $K(K+1) / 2$ vector made from $\left[\mathbf{R}_{s}\right]_{i, j}$ for $1 \leq$ $i \leq j \leq K$. In the particular case, where prior knowledge of uncorrelated or full coherent sources $s_{t, k}$ are incorporated, the parameter $\boldsymbol{\rho}$ reduces to $\boldsymbol{\rho}=\left(\sigma_{1}^{2}, . ., \sigma_{k}^{2}, . ., \sigma_{K}^{2}\right)^{T}$ where $\sigma_{k}^{2} \stackrel{\text { def }}{=} \mathrm{E}\left(s_{t, k}^{2}\right)=\left[\mathbf{R}_{s}\right]_{k, k}$ and to $\boldsymbol{\rho}=\mathbf{c}=\left(c_{1}, \ldots, c_{K}\right)^{T}$ where $\mathbf{R}_{s}=\mathbf{c c}^{T}$, respectively.

Under the stochastic assumption, $\left(\mathbf{y}_{t}\right)_{t=1, . ., T}$ are independent and noncircular Gaussian distributed and therefore the Fisher information matrix (FIM) for the parameter $\boldsymbol{\alpha}$ is given (elementwise) by [7]:

$$
\operatorname{FIM}_{i, j}=\frac{T}{2} \operatorname{Tr}\left[\frac{\partial \mathbf{R}_{\tilde{y}}}{\partial \alpha_{i}} \mathbf{R}_{\tilde{y}}^{-1} \frac{\partial \mathbf{R}_{\tilde{y}}}{\partial \alpha_{j}} \mathbf{R}_{\tilde{y}}^{-1}\right],
$$

where $\mathbf{R}_{\tilde{y}}$ is the covariance of the extended signal $\widetilde{\mathbf{y}}_{t} \stackrel{\text { def }}{=}$ $\left[\mathbf{y}_{t}^{T}, \mathbf{y}_{t}^{H}\right]^{T}$ given by:

$$
\mathbf{R}_{\tilde{y}} \stackrel{\text { def }}{=} \mathrm{E}\left(\widetilde{\mathbf{y}}_{t} \widetilde{\mathbf{y}}_{t}^{H}\right)=\widetilde{\mathbf{A}} \mathbf{R}_{s} \widetilde{\mathbf{A}}^{H}+\sigma_{n}^{2} \mathbf{I},
$$

where $\widetilde{\mathbf{A}} \stackrel{\text { def }}{=}\left[\begin{array}{c}\mathbf{A}_{\theta} \boldsymbol{\Delta}_{\phi} \\ \mathbf{A}_{\theta}^{*} \boldsymbol{\Delta}_{\phi}^{*}\end{array}\right]=\left[\widetilde{\mathbf{a}}_{1}, \ldots, \widetilde{\mathbf{a}}_{K}\right]$ with $\widetilde{\mathbf{a}}_{k} \stackrel{\text { def }}{=}$ $\left[\mathbf{a}^{T}\left(\theta_{k}\right) e^{i \phi_{k}}, \mathbf{a}^{H}\left(\theta_{k}\right) e^{-i \phi_{k}}\right]^{T}$.

The purpose of the next section is to directly derive the SCRB of the parameter $\theta$ alone from the FIM (6). Noting that the parameters $\theta_{k}$ and $\phi_{k}$ are non-linearly related in the extended steering vector $\widetilde{\mathbf{a}}_{k}$, closed-form expressions of the SCRB of the couple $\boldsymbol{\omega} \stackrel{\text { def }}{=}\left(\boldsymbol{\theta}^{T}, \phi^{T}\right)^{T}$ are first derived through its inverse $\left[\mathrm{CRB}_{\text {sto }}(\boldsymbol{\omega})\right]^{-1} \stackrel{\text { def }}{=}\left[\begin{array}{ll}\mathbf{I}_{\boldsymbol{\theta}, \boldsymbol{\theta}} & \mathbf{I}_{\boldsymbol{\theta}, \boldsymbol{\phi}} \\ \mathbf{I}_{\boldsymbol{\theta}, \boldsymbol{\phi}}^{T} & \mathbf{I}_{\boldsymbol{\phi}, \boldsymbol{\phi}}\end{array}\right]$. Thus the SCRB of $\boldsymbol{\theta}$ alone is deduced by

$$
\mathrm{CRB}_{\text {sto }}(\boldsymbol{\theta})=\left(\mathbf{I}_{\boldsymbol{\theta}, \boldsymbol{\theta}}-\mathbf{I}_{\boldsymbol{\theta}, \boldsymbol{\phi}} \mathbf{I}_{\boldsymbol{\phi}, \boldsymbol{\phi}}^{-1} \mathbf{I}_{\boldsymbol{\theta}, \boldsymbol{\phi}}^{T}\right)^{-1} .
$$

\section{DERIVATION OF THE DIFFERENT CRB}

Writing the FIM (6) in compact matrix from as:

$$
\mathrm{FIM}=\frac{T}{2}\left(\frac{\partial \mathbf{r}_{\tilde{y}}}{\partial \boldsymbol{\alpha}^{T}}\right)^{H}\left(\mathbf{R}_{\tilde{y}}^{-T} \otimes \mathbf{R}_{\tilde{y}}^{-1}\right)\left(\frac{\partial \mathbf{r}_{\tilde{y}}}{\partial \boldsymbol{\alpha}^{T}}\right),
$$

where $\mathbf{r}_{\tilde{y}} \stackrel{\text { def }}{=} \operatorname{vec}\left(\mathbf{R}_{\tilde{y}}\right)=\left(\widetilde{\mathbf{A}}^{*} \otimes \widetilde{\mathbf{A}}\right) \operatorname{vec}\left(\mathbf{R}_{s}\right)+\sigma_{n}^{2} \operatorname{vec}(\mathbf{I})$ (with $\otimes$ is the Kronecker product), all the first steps of the proof given in [16] apply. In particular, using the partition $\left(\mathbf{R}_{\tilde{y}}^{-T / 2} \otimes \mathbf{R}_{\tilde{y}}^{-1 / 2}\right)\left(\frac{\partial \mathbf{r}_{\tilde{y}}}{\partial \boldsymbol{\omega}^{T}} \mid \frac{\partial \mathbf{r}_{\tilde{y}}}{\partial \boldsymbol{\rho}^{T}}, \frac{\partial \mathbf{r}_{\tilde{y}}}{\partial \sigma_{n}^{2}}\right) \stackrel{\text { def }}{=}(\mathbf{G} \mid \mathbf{V} \mathbf{u})$, we can deduce from (9):

$$
\frac{2}{T}\left[\mathrm{CRB}_{\mathrm{sto}}(\boldsymbol{\omega})\right]^{-1}=\mathbf{G}^{H} \mathbf{\Pi}_{\Delta}^{\perp} \mathbf{G} .
$$

with $\boldsymbol{\Delta} \stackrel{\text { def }}{=}(\mathbf{V} \mathbf{u})$ and $\boldsymbol{\Pi}_{\Delta}^{\perp} \stackrel{\text { def }}{=} \mathbf{I}-\boldsymbol{\Pi}_{\boldsymbol{\Delta}}$ where $\boldsymbol{\Pi}_{\boldsymbol{\Delta}}$ denotes the orthonormal projector on the columns of $\boldsymbol{\Delta}$. Starting from (10), where $\Pi_{\Delta}^{\perp}$ is given by [16, rel. (14], the main steps of the proof of the following theorem are given in the Appendix:

Theorem 1: The SCRB under the general rectilinear assumption is given by for $K<2 M$ :

$$
\operatorname{CRB}_{\text {sto }}^{\text {rec }_{1}}(\boldsymbol{\omega})=\frac{\sigma_{n}^{2}}{T}\left(\left(\widetilde{\mathbf{D}}_{\omega}^{H} \mathbf{\Pi}_{\widetilde{\mathbf{A}}}^{\perp} \widetilde{\mathbf{D}}_{\omega}\right) \odot\left(\left(\begin{array}{ll}
1 & 1 \\
1 & 1
\end{array}\right) \otimes \widetilde{\mathbf{H}}\right)\right)^{-1}
$$

with $\boldsymbol{\Pi}_{\widetilde{\mathbf{A}}}^{\perp} \stackrel{\text { def }}{=} \mathbf{I}-\boldsymbol{\Pi}_{\widetilde{\mathbf{A}}}$, where $\boldsymbol{\Pi}_{\widetilde{\mathbf{A}}}$ denotes the orthonormal projector on the columns of $\widetilde{\mathbf{A}}, \widetilde{\mathbf{D}}_{\omega} \stackrel{\text { def }}{=}\left[\widetilde{\mathbf{D}}_{\theta}, \widetilde{\mathbf{D}}_{\phi}\right]$ with $\widetilde{\mathbf{D}}_{\theta} \stackrel{\text { def }}{=}\left[\frac{\partial \tilde{\mathbf{a}}_{1}}{\partial \theta_{1}}, \ldots, \frac{\partial \tilde{\mathbf{a}}_{K}}{\partial \theta_{K}}\right], \widetilde{\mathbf{D}}_{\phi} \stackrel{\text { def }}{=}\left[\frac{\partial \tilde{\mathbf{a}}_{1}}{\partial \phi_{1}}, \ldots, \frac{\partial \tilde{\mathbf{a}}_{K}}{\partial \phi_{K}}\right], \widetilde{\mathbf{H}} \stackrel{\text { def }}{=}$ $\mathbf{R}_{s} \widetilde{\mathbf{A}}^{H} \mathbf{R}_{\tilde{y}}^{-1} \widetilde{\mathbf{A}} \mathbf{R}_{s}$ and $\odot$ denotes the element by element matrix product. Applying (8), the SCRB on $\boldsymbol{\theta}$ alone is given by:

$$
\begin{aligned}
& \operatorname{CRB}_{\text {sto }}^{\text {rec }}((\boldsymbol{\theta})=\frac{\sigma_{n}^{2}}{T}\left(\left[\left(\widetilde{\mathbf{D}}_{\theta}^{H} \boldsymbol{\Pi}_{\widetilde{\mathbf{A}}}^{\perp} \widetilde{\mathbf{D}}_{\theta}\right) \odot \widetilde{\mathbf{H}}\right]-\left[\left(\widetilde{\mathbf{D}}_{\theta}^{H} \boldsymbol{\Pi}_{\widetilde{\mathbf{A}}}^{\perp} \widetilde{\mathbf{D}}_{\phi}\right) \odot \widetilde{\mathbf{H}}\right]\right. \\
&\left.\times\left[\left(\widetilde{\mathbf{D}}_{\phi}^{H} \boldsymbol{\Pi}_{\widetilde{\mathbf{A}}}^{\perp} \widetilde{\mathbf{D}}_{\phi}\right) \odot \widetilde{\mathbf{H}}\right]^{-1}\left[\left(\widetilde{\mathbf{D}}_{\phi}^{H} \boldsymbol{\Pi}_{\widetilde{\mathbf{A}}}^{\perp} \widetilde{\mathbf{D}}_{\theta}\right) \odot \widetilde{\mathbf{H}}\right]\right) \cdot(12)
\end{aligned}
$$

Including the prior knowledge that the $K$ rectilinear sources are coherent (where $\mathbf{R}_{s}=\mathbf{c c}^{T}$ ), which appears in specular multipath propagation, the main steps of the proof of the following theorem are given in the Appendix:

Theorem 2: The SCRB under the prior knowledge of fully coherent rectilinear sources is given by for $K<2 M$ :

$$
\mathrm{CRB}_{\mathrm{sto}}^{\mathrm{rec}_{2}}(\boldsymbol{\omega})=\frac{\sigma_{n}^{2}}{T \kappa_{c}}\left(\left(\widetilde{\mathbf{D}}_{\omega}^{H} \boldsymbol{\Pi}_{\widetilde{\mathbf{A}}}^{\perp} \widetilde{\mathbf{D}}_{\omega}\right) \odot\left(\left(\begin{array}{ll}
1 & 1 \\
1 & 1
\end{array}\right) \otimes \mathbf{R}_{s}\right)\right)^{-1}
$$

where $\kappa_{c} \stackrel{\text { def }}{=} \mathbf{c}^{T} \widetilde{\mathbf{A}}^{H} \mathbf{R}_{\tilde{y}}^{-1} \widetilde{\mathbf{A}} \mathbf{c}$.

Including now the prior knowledge that the $K$ rectilinear sources are uncorrelated, the following theorem (presented in [9]) is proved in the Appendix:

Theorem 3: The SCRB under the prior knowledge of uncorrelated rectilinear sources is given by $K<2 M$ :

$$
\mathrm{CRB}_{\mathrm{sto}}^{\mathrm{rec}_{3}}(\boldsymbol{\omega})=\frac{2}{T}\left(\widetilde{\boldsymbol{\Delta}}_{\sigma} \overline{\mathbf{D}}_{\omega}^{H} \widetilde{\mathbf{B}}\left(\widetilde{\mathbf{B}}^{H} \overline{\mathbf{G}} \widetilde{\mathbf{B}}\right)^{-1} \widetilde{\mathbf{B}}^{H} \overline{\mathbf{D}}_{\omega} \widetilde{\boldsymbol{\Delta}}_{\sigma}\right)^{-1}
$$

where $\overline{\mathbf{G}} \stackrel{\text { def }}{=} \mathbf{R}_{\tilde{y}}^{T} \otimes \mathbf{R}_{\tilde{y}}+\frac{\sigma_{n}^{4}}{2 M-K} \operatorname{vec}\left(\boldsymbol{\Pi}_{\widetilde{\mathbf{A}}}\right) \operatorname{vec}^{H}\left(\boldsymbol{\Pi}_{\widetilde{\mathbf{A}}}\right), \widetilde{\boldsymbol{\Delta}}_{\sigma} \stackrel{\text { def }}{=}$ $\operatorname{Diag}\left(\sigma_{1}^{2}, \ldots, \sigma_{K}^{2}, \sigma_{1}^{2}, \ldots, \sigma_{K}^{2}\right), \overline{\mathbf{D}}_{\omega} \stackrel{\text { def }}{=}\left(\widetilde{\mathbf{A}}^{*} \mid \widetilde{\mathbf{A}}^{*}\right) \circ\left(\widetilde{\mathbf{D}}_{\theta} \mid \widetilde{\mathbf{D}}_{\phi}\right)+$ $\left(\widetilde{\mathbf{D}}_{\theta}^{*} \mid \widetilde{\mathbf{D}}_{\phi}^{*}\right) \circ(\widetilde{\mathbf{A}} \mid \widetilde{\mathbf{A}}), \widetilde{\mathbf{B}}$ is any $(2 M)^{2} \times\left((2 M)^{2}-K\right)$ matrix whose columns span the null space of $\widetilde{\mathbf{A}}^{*} \circ \widetilde{\mathbf{A}}$, where $\circ$ is the columnwise Kronecker product.

Finally, to make comparisons with the DCRB, we recall its closed-form expression derived in [9] and [10] and then in [11] under more general rectilinear models for $K<2 M$.

$$
\operatorname{CRB}_{\mathrm{det}}^{\mathrm{rec}}(\boldsymbol{\omega})=\frac{\sigma_{n}^{2}}{T}\left(\left(\widetilde{\mathbf{D}}_{\omega}^{H} \mathbf{\Pi}_{\widetilde{\mathbf{A}}}^{\perp} \widetilde{\mathbf{D}}_{\omega}\right) \odot\left(\left(\begin{array}{ll}
1 & 1 \\
1 & 1
\end{array}\right) \otimes \mathbf{R}_{s, T}\right)\right)^{-1}
$$

where $\mathbf{R}_{s, T} \stackrel{\text { def }}{=} \frac{1}{T} \sum_{t=1}^{T} \mathbf{s}_{t} \mathbf{s}_{t}^{T}$. We also recall the closedform expression of the SCRB derived under the assumption of arbitrary noncircular sources in [7], applied to rectilinear 
sources for $K<M$ :

$$
\mathrm{CRB}_{\text {sto }}^{\mathrm{nc}}(\boldsymbol{\theta})=\frac{\sigma_{n}^{2}}{2 T}\left(\operatorname{Re}\left[\left(\mathbf{D}_{\theta}^{H} \boldsymbol{\Pi}_{\mathbf{A}_{\theta}}^{\perp} \mathbf{D}_{\theta}\right) \odot\left(\boldsymbol{\Delta}_{\phi}^{*} \widetilde{\mathbf{H}} \boldsymbol{\Delta}_{\phi}\right)\right]\right)^{-1}
$$

where $\mathbf{D}_{\theta} \stackrel{\text { def }}{=}\left[\frac{\partial \mathbf{a}_{1}}{\partial \theta_{1}}, \ldots, \frac{\partial \mathbf{a}_{K}}{\partial \theta_{K}}\right]$.

\section{ANALYTICAL AND NUMERICAL COMPARISONS}

Considering the comparison of the previously introduced closed-form expressions of the CRB, the main steps of the proof of the following theorem are given in the Appendix:

Theorem 4: Under the general rectilinear assumption, the DCRB (for $T \rightarrow \infty$, i.e., replacing $\mathbf{R}_{s, T}$ by $\mathbf{R}_{s}$ ) and SCRB have the relationships:

$$
\operatorname{CRB}_{\text {det }}^{\text {rec }}(\boldsymbol{\theta}) \leq \operatorname{CRB}_{\text {sto }}^{\text {rec }_{1}}(\boldsymbol{\theta}) \leq \operatorname{CRB}_{\text {sto }}^{\text {nc }}(\boldsymbol{\theta}) .
$$

Note that for a finite value of $T$, we cannot be sure that $\mathrm{CRB}_{\text {det }}^{\text {rec }}(\boldsymbol{\theta}) \leq \operatorname{CRB}_{\text {sto }}^{\text {rec }_{1}}(\boldsymbol{\theta})$. In fact for low $T$ and high SNRs, the inequality reverses.

If we consider now the exploitation of prior knowledge, the following theorem is proved in the Appendix:

Theorem 5: Under the prior knowledge that the sources are rectilinear uncorrelated, $\mathrm{CRB}_{\text {sto }}^{\mathrm{rec}_{3}}(\boldsymbol{\theta})$ is reduced w.r.t. $\mathrm{CRB}_{\text {sto }}^{\text {rec }}(\boldsymbol{\theta})$. In contrast, the exploitation of fully coherency of the sources does not reduce $\operatorname{CRB}_{\text {sto }}^{\text {rec }_{1}}(\boldsymbol{\theta})$ :

$$
\mathrm{CRB}_{\text {sto }}^{\mathrm{rec}_{3}}(\boldsymbol{\theta}) \leq \mathrm{CRB}_{\text {sto }}^{\mathrm{rec}_{1}}(\boldsymbol{\theta}), \mathrm{CRB}_{\text {sto }}^{\mathrm{rec}_{2}}(\boldsymbol{\theta})=\mathrm{CRB}_{\text {sto }}^{\mathrm{rec}_{1}}(\boldsymbol{\theta}) .
$$

Note that similar properties have been proved for circular sources in [17] for uncorrelated sources and in [18] for fully coherent sources.

Finally, in the case of a single rectilinear source, we have proved after tedious algebraic manipulations that the SCRBs of $\theta_{1}$ alone deduced from (11), (13) and (14) reduce to:

$$
\operatorname{CRB}_{\text {sto }}^{\text {rec }}\left(\theta_{1}\right)=\frac{1}{2 T \mathbf{a}_{1}^{\prime H} \Pi_{\mathbf{a}_{1}}^{\perp} \mathbf{a}_{1}^{\prime}} \frac{\sigma_{n}^{2}}{\sigma_{s}^{2}}\left(1+\frac{\sigma_{n}^{2}}{2 \sigma_{s}^{2}\left\|\mathbf{a}_{1}\right\|^{2}}\right),
$$

where $\mathbf{a}_{1} \stackrel{\text { def }}{=} \mathbf{a}\left(\theta_{1}\right)$ and $\mathbf{a}_{1}^{\prime} \stackrel{\text { def }}{=} d \mathbf{a}\left(\theta_{1}\right) / d \theta_{1}$. Comparing (19) to the SCRB derived in [7] under the general noncircular assumption, we see that $\operatorname{CRB}_{\text {sto }}^{\text {rec }}\left(\theta_{1}\right)=\operatorname{CRB}_{\text {sto }}^{\text {nc }}\left(\theta_{1}\right)$, i.e., the SCRB is not reduced by exploiting the rectilinear prior knowledge.

To illustrate, the difference between the different CRBs, we consider now the case of two equal-power rectilinear sources of signal-to-noise ratio $10 \log _{10}\left(\sigma_{s}^{2} / \sigma_{n}^{2}\right)=10 \mathrm{~dB}$ and correlation $\rho$, impinging on an ULA of $M=6$ sensors with half-wavelength spacing. Figs 1-3 exhibit different ratios of CRB. Fig.1 shows that there are significant gaps between the DSCB and the SCRB for closely spaced and strongly correlated rectilinear sources. More generally, extensive numerical comparisons have shown that this gap increases for low SNR, low phase and DOA separations and high source correlation, but this gap will always vanish for high SNR. This proves that the conclusions based on the DCRB may be very optimistic for not too high
SNR. Fig. 2 highlights that the exploitation of the prior of rectilinearity greatly reduces the estimation error for closely spaced and uncorrelated sources. Finally, Fig. 3 proves that the joint exploitation of the prior of uncorrelatedness and rectilinearity greatly reduces the estimation error for closely spaced sources with different phases.

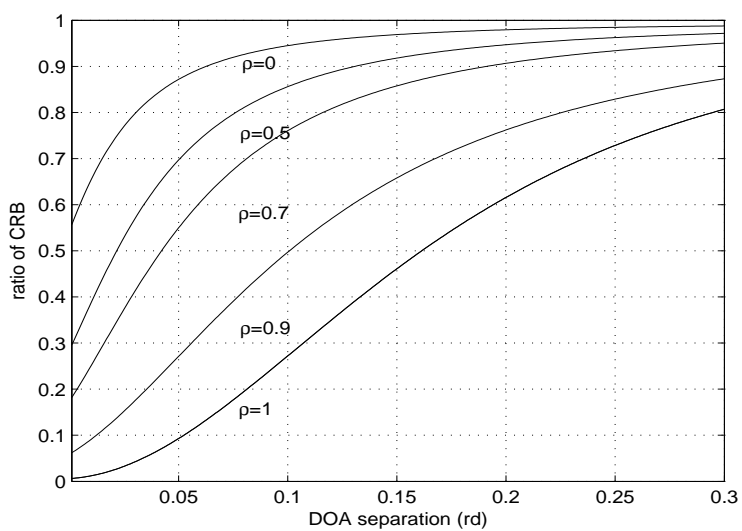

Fig. 1. Ratio $\mathrm{CRB}_{\text {det }}^{\mathrm{rec}}\left(\theta_{1}\right) / \mathrm{CRB}_{\text {sto }}^{\mathrm{rec}}\left(\theta_{1}\right)$ for $\phi_{1}-\phi_{2}=0.1 \mathrm{rd}$.

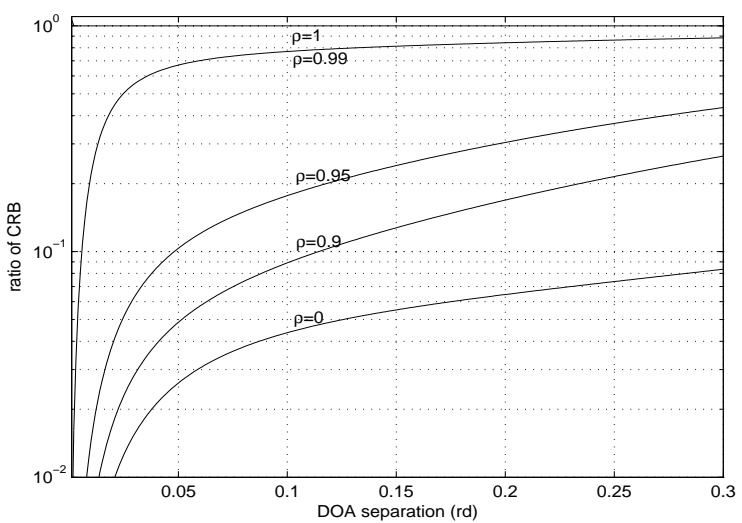

Fig. 2. Ratio $\operatorname{CRB}_{\text {sto }}^{\text {rec }}\left(\theta_{1}\right) / \operatorname{CRB}_{\text {sto }}^{\text {nc }}\left(\theta_{1}\right)$ for $\phi_{1}-\phi_{2}=0.1 \mathrm{rd}$.

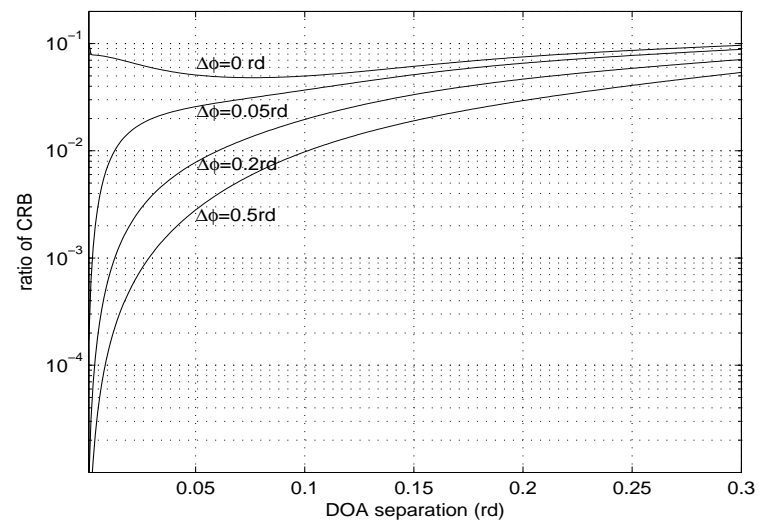

Fig. 3. Ratio $\operatorname{CRB}_{\text {sto }}^{\mathrm{rec}_{3}}\left(\theta_{1}\right) / \mathrm{CRB}_{\text {sto }}^{\text {nc }}\left(\theta_{1}\right)$ for $\rho=0$.

\section{APPENDIX}

Proof of theorem 1: (Detailed proofs of Theorem 1 are available at [23]) Since $\mathbf{R}_{s}$ is a $(K \times K)$ real symmetric matrix, it then follows from [19, rel.(7.18)] that $\operatorname{vec}\left(\mathbf{R}_{s}\right)=\mathbf{D}_{K} \boldsymbol{\rho}$ 
where $\mathbf{D}_{K}$ is a so-called duplication matrix, and hence [16, rel.(19)] becomes

$$
\mathbf{V}=\left(\mathbf{R}_{\tilde{y}}^{-T / 2} \tilde{\mathbf{A}}^{*} \otimes \mathbf{R}_{\tilde{y}}^{-1 / 2} \tilde{\mathbf{A}}\right) \mathbf{D}_{K} \stackrel{\text { def }}{=} \mathbf{W} \mathbf{D}_{K} .
$$

Then it follows from [19, Theorem 7.38], and some simple algebraic manipulations using [19, Theorem 7.34, rel.(b)] that $[16$, rel.(20)] becomes

$$
\mathbf{\Pi}_{\mathbf{V}}^{\perp}=\mathbf{I}-\mathbf{W}(\mathbf{U} \otimes \mathbf{U}) \mathbf{N}_{K} \mathbf{W}^{H},
$$

where $\mathbf{U} \stackrel{\text { def }}{=} \tilde{\mathbf{A}}^{H} \mathbf{R}_{\tilde{y}}^{-1} \tilde{\mathbf{A}}$ and $\mathbf{N}_{K}$ is an $(K \times K)$ matrix defined in [19, Theorem 7.34]. By evaluating the derivatives in $\mathbf{G}$ and $\mathbf{u}$, and through some further algebra, one finds

$$
\mathbf{u}^{H} \boldsymbol{\Pi}_{\mathbf{V}}^{\perp} \mathbf{g}_{k}=0
$$

where $\mathbf{g}_{k}$ is the $k$ th column of $\mathbf{G}$ given by $\mathbf{g}_{k}=\operatorname{vec}\left(\mathbf{Z}_{k}+\right.$ $\left.\mathbf{Z}_{k}^{H}\right)$ and where $\mathbf{Z}_{k} \stackrel{\text { def }}{=} \mathbf{R}_{\tilde{y}}^{-1 / 2} \tilde{\mathbf{A}} \mathbf{r}_{s, k} \tilde{\mathbf{a}}_{k}^{\prime H} \mathbf{R}_{\tilde{y}}^{-1 / 2}, \tilde{\mathbf{a}}_{k}^{\prime} \stackrel{\text { def }}{=}$ $d \tilde{\mathbf{a}}_{k} / d w_{k}$ and $\mathbf{r}_{s, k}$ is the kth column of $\mathbf{R}_{s}$. This identity allows us to rewrite the individual elements of (10) as

$$
\frac{2}{T}\left[\mathrm{CRB}_{\text {sto }}^{-1}(\boldsymbol{\omega})\right]_{k, l}=\mathbf{g}_{k}^{H} \mathbf{\Pi}_{\mathbf{V}}^{\perp} \mathbf{g}_{l} .
$$

By further calculations we get

$$
\left[\mathrm{CRB}_{\text {sto }}^{-1}(\boldsymbol{\omega})\right]_{k, l}=\frac{T}{\sigma_{n}^{2}} \operatorname{Re}\left(\left(\tilde{\mathbf{a}}_{k}^{\prime H} \boldsymbol{\Pi}_{\tilde{\mathbf{A}}}^{\perp} \tilde{\mathbf{a}}_{l}^{\prime}\right)\left(\mathbf{r}_{s, k}^{T} \tilde{\mathbf{A}}^{H} \mathbf{R}_{\tilde{y}}^{-1} \tilde{\mathbf{A}} \mathbf{r}_{s, l}\right)\right) \text {. }
$$

Finally, we can write (20) in matrix form as in (11).

Proof of theorem 2: (Detailed proofs of Theorem 1 are available at [23]) We follow the steps similar to those in the proof of theorem 1. Since $\mathbf{R}_{s}=\mathbf{c c}^{T}$ and its derivative w.r.t. $\mathbf{c}$ is given by $\mathbf{D}_{c} \stackrel{\text { def }}{=} \mathbf{c} \otimes \mathbf{I}+\mathbf{I} \otimes \mathbf{c}=2 \mathbf{N}_{K}(\mathbf{c} \otimes \mathbf{I})$, it follows then that $\mathbf{V}$ has the form $\mathbf{V}=\mathbf{W D}_{c}$. After some algebraic manipulation using [19, Theorem 7.34, rel.(d)], it follows that

$$
\boldsymbol{\Pi}_{\mathbf{V}}^{\perp} \stackrel{\text { def }}{=} \mathbf{I}-\mathbf{V}\left(\mathbf{V}^{H} \mathbf{V}\right)^{-1} \mathbf{V}^{H}=\mathbf{I}-\mathbf{V}_{1} \overline{\mathbf{V}}^{-1} \mathbf{V}_{1}^{H}
$$

with $\mathbf{V}_{1} \stackrel{\text { def }}{=} \mathbf{W N}_{K}(\mathbf{c} \otimes \mathbf{I})$ and $\overline{\mathbf{V}} \stackrel{\text { def }}{=} \frac{1}{2}\left(\kappa_{c} \mathbf{U}+\mathbf{u}_{c} \mathbf{u}_{c}^{T}\right)$ where $\mathbf{u}_{c} \stackrel{\text { def }}{=} \mathbf{U} \mathbf{c}$ and $\kappa_{c} \stackrel{\text { def }}{=} \mathbf{c}^{T} \mathbf{U} \mathbf{c}$. Thanks to the matrix inversion lemma, we have $\overline{\mathbf{V}}^{-1}=\frac{2}{\kappa_{c}}\left(\mathbf{U}^{-1}-\frac{1}{2 \kappa_{c}} \mathbf{c c}^{T}\right)$. Through some further algebra using $\mathbf{u}=\operatorname{vec}\left(\mathbf{R}_{\tilde{y}}^{-1}\right)$ and $\mathbf{g}_{k}=\operatorname{vec}\left(\mathbf{Z}_{k}+\mathbf{Z}_{k}^{H}\right)$ where $\mathbf{Z}_{k} \stackrel{\text { def }}{=} \mathbf{R}_{\tilde{y}}^{-1 / 2} \tilde{\mathbf{A}} \mathbf{c} \tilde{\mathbf{a}}_{k}^{\prime H} \mathbf{R}_{\tilde{y}}^{-1 / 2}$, one finds that $\mathbf{u}^{H} \mathbf{V}_{1}=\mathbf{c}^{T} \overline{\mathbf{U}}$ where $\overline{\mathbf{U}} \stackrel{\text { def }}{=} \tilde{\mathbf{A}}^{H} \mathbf{R}_{\tilde{y}}^{-2} \tilde{\mathbf{A}}, \mathbf{u}^{H} \mathbf{g}_{k}=$ $2 c_{k} \tilde{\mathbf{a}}_{k}^{\prime H} \mathbf{R}_{\tilde{y}}^{-2} \tilde{\mathbf{A}} \mathbf{c}$ and $\mathbf{V}_{1}^{H} \mathbf{g}_{k}=c_{k}\left(\nu_{c}^{(k)} \mathbf{u}_{c}+\kappa_{c} \tilde{\mathbf{A}}^{H} \mathbf{R}_{\tilde{y}}^{-1} \tilde{\mathbf{a}}_{k}^{\prime}\right)$ where $\nu_{c}^{(k)} \stackrel{\text { def }}{=} \tilde{\mathbf{a}}_{k}^{\prime H} \mathbf{R}_{\tilde{y}}^{-1} \tilde{\mathbf{A}} \mathbf{c}$. By further calculations we arrive at $\mathbf{u}^{H} \boldsymbol{\Pi}_{\mathbf{V}}^{\perp} \mathbf{g}_{k}=0$. This identity allows us to rewrite the individual elements of (10) as

$$
\frac{2}{T}\left[\mathrm{CRB}_{\text {sto }}^{-1}(\boldsymbol{\omega})\right]_{k, l}=\mathbf{g}_{k}^{H} \boldsymbol{\Pi}_{\mathbf{V}}^{\perp} \mathbf{g}_{l}=\frac{2 \kappa_{c} T}{\sigma_{n}^{2}} c_{k} c_{l}\left(\tilde{\mathbf{a}}_{k}^{\prime H} \boldsymbol{\Pi}_{\tilde{\mathbf{A}}}^{\perp} \tilde{\mathbf{a}}_{l}^{\prime}\right),
$$

which can also be written in the matrix form (13).

Proof of theorem 3: Noting that (7) becomes $\mathbf{R}_{\tilde{y}}=$ $\sum_{k=1}^{K} \sigma_{k}^{2} \widetilde{\mathbf{a}}_{k} \widetilde{\mathbf{a}}_{k}^{H}+\sigma_{n}^{2} \mathbf{I}$, all the steps of the proof given in [17, Appendix A] apply to the parameter $\boldsymbol{\omega}$ with the
FIM (6) associated with the noncircular zero-mean Gaussian distribution of $\mathbf{y}_{t}$.

Proof of theorem 4: Using $\mathbf{R}_{s} \geq \mathbf{R}_{s} \widetilde{\mathbf{A}}^{H} \mathbf{R}_{\tilde{y}}^{-1} \widetilde{\mathbf{A}} \mathbf{R}_{s}$ thanks to [20, rel. B.6.37] and noting that $\left(\begin{array}{ll}1 & 1 \\ 1 & 1\end{array}\right)$ is positive semidefinite, we have $\left(\begin{array}{ll}1 & 1 \\ 1 & 1\end{array}\right) \otimes\left(\mathbf{R}_{s}-\widetilde{\mathbf{H}}\right) \geq 0$ from [19, th. 7.10]. Then, noting that $\widetilde{\mathbf{D}}_{\omega}^{H} \mathbf{\Pi} \widetilde{\mathbf{A}}_{\widetilde{\mathbf{D}}}^{\perp} \widetilde{\mathbf{D}}_{\omega}$ is positive definite, we have $\left(\widetilde{\mathbf{D}}_{\omega}^{H} \boldsymbol{\Pi}_{\widetilde{\mathbf{A}}}^{\perp} \widetilde{\mathbf{D}}_{\omega}\right) \odot\left(\left(\begin{array}{ll}1 & 1 \\ 1 & 1\end{array}\right) \otimes\left(\mathbf{R}_{s}-\widetilde{\mathbf{H}}\right)\right) \geq 0$ thanks to [20, rel. R.19, p.358], and $\operatorname{CRB}_{\text {det }}^{\text {rec }}(\boldsymbol{\theta}) \leq \operatorname{CRB}_{\text {sto }}^{\text {rec }}(\boldsymbol{\theta})$ directly follows.

Consider the parametrization $\boldsymbol{\alpha}_{\mathrm{nc}}=\left(\boldsymbol{\theta}^{T}, \boldsymbol{\rho}_{\mathrm{nc}}^{T}, \sigma_{n}^{2}\right)^{T}$ associated with the assumption of arbitrary noncircular sources in [7], where $\boldsymbol{\rho}_{\mathrm{nc}}$ is the $2 K^{2}+K$ vector made from $\left[\operatorname{Re}\left(\mathbf{R}_{x}\right)\right]_{i, j},\left[\operatorname{Im}\left(\mathbf{R}_{x}\right)\right]_{i, j},\left[\operatorname{Re}\left(\mathbf{C}_{x}\right)\right]_{i, j}$ and $\left[\operatorname{Re}\left(\mathbf{C}_{x}\right)\right]_{i, j}$ for $1 \leq i<j \leq K$, and $\left[\mathbf{R}_{x}\right]_{i, i}, \quad\left[\operatorname{Re}\left(\mathbf{C}_{x}\right)\right]_{i, i}$ and $\left[\operatorname{Im}\left(\mathbf{C}_{x}\right)\right]_{i, i}$ for $1 \leq i \leq K$, where $\mathbf{R}_{x} \stackrel{\text { def }}{=} \mathrm{E}\left(\mathbf{x}_{t} \mathbf{x}_{t}^{H}\right)$ and $\mathbf{C}_{x} \stackrel{\text { def }}{=} \mathrm{E}\left(\mathbf{x}_{t} \mathbf{x}_{t}^{T}\right)$ with $\mathbf{x}_{t} \stackrel{\text { def }}{=}\left(x_{t, 1}, \ldots, x_{t, K}\right)^{T}$. Consider now the one to one mapping between $\boldsymbol{\alpha}_{\mathrm{nc}}$ and $\boldsymbol{\alpha}_{\mathrm{nc}}^{\prime}=$ $\left(\boldsymbol{\theta}^{T}, \boldsymbol{\phi}^{T}, \boldsymbol{\rho}_{1}^{T}, \boldsymbol{\rho}^{\prime T}, \sigma_{n}^{2}\right)^{T}$ where $\boldsymbol{\phi}=\left(\phi_{1}, . ., \phi_{k}, . ., \phi_{K}\right)^{T}$ is defined by $2 \phi_{k} \stackrel{\text { def }}{=} \angle\left[\mathbf{C}_{x}\right]_{k, k} /\left[\mathbf{R}_{x}\right]_{k, k}, \boldsymbol{\rho}_{1}$ is the vector made from $\left[\operatorname{Re}\left(\mathbf{R}_{s}\right)\right]_{i, j}$ for $1 \leq i \leq j \leq K$, and $\boldsymbol{\rho}^{\prime}$ is the vector gathering $\left[\operatorname{Im}\left(\mathbf{R}_{s}\right)\right]_{i, j},\left[\operatorname{Im}\left(\mathbf{C}_{s}\right)\right]_{i, j}$ for $1 \leq i<j \leq K$ and $\left[\operatorname{Re}\left(\mathbf{C}_{s}\right)\right]_{i, j}$ for $1 \leq i \leq j \leq K$.

Let FIM $_{1}$, FIM $_{\text {nc }}$ and FIM nc $^{\prime}$ be the FIM associated with the stochastic parametrizations (3), $\boldsymbol{\alpha}_{\mathrm{nc}}$ and $\boldsymbol{\alpha}_{\mathrm{nc}}^{\prime}$, respectively. From the one to one mapping $\boldsymbol{\alpha}_{\mathrm{nc}} \leftrightarrow \boldsymbol{\alpha}_{\mathrm{nc}}^{\prime}$, we get $\left[\mathrm{FIM}_{\mathrm{nc}}^{\prime}\right]_{\theta}=\left[\mathrm{FIM}_{\mathrm{nc}}\right]_{\theta}$ (where $[.]_{\theta}$ denotes the submatrix determined by the first $K$ rows and columns). Furthermore $\mathrm{FIM}_{1}=\left[\mathrm{FIM}^{\prime}{ }_{\mathrm{nc}}\right]_{1}$ where $[.]_{1}$ denotes the principal submatrix determined by the rows and columns induced by the parametrization of $\mathrm{FIM}_{1}$. Consequently, taking the inverse, we get $\left[\mathrm{FIM}_{1}\right]^{-1} \leq\left[\mathrm{FIM}_{\mathrm{nc}}^{\prime-1}\right]_{1}[22$, th. 7.7 .8$]$. Then taking the principal submatrix determined by the first $K$ rows and columns, we get $\mathrm{CRB}_{\text {sto }}^{\mathrm{rec}_{1}}(\boldsymbol{\theta}) \leq \mathrm{CRB}_{\text {sto }}^{\text {nc }}(\boldsymbol{\theta})$.

Proof of theorem 5: Let FIM 3 be the FIM associated with the parametrizations (3) for which $\boldsymbol{\rho}=\left(\left[\mathbf{R}_{s}\right]_{k, k ; 1 \leq k \leq K}\right)^{T}$. For rectilinear uncorrelated sources, $\mathrm{FIM}_{3}=\left[\mathrm{FIM}_{1}\right]_{3}$, where $[.]_{3}$ denotes the principal submatrix determined by the rows and columns induced by the parametrization of $\mathrm{FIM}_{3}$. Taking the inverse, we get $\left[\mathrm{FIM}_{3}\right]^{-1} \leq\left[\mathrm{FIM}_{1}^{-1}\right]_{3}[22$, th. 7.7.8]. Then taking the principal submatrix determined by the first $K$ rows and columns, we get $\operatorname{CRB}_{\text {sto }}^{\mathrm{rec}_{3}}(\boldsymbol{\theta}) \leq \mathrm{CRB}_{\text {sto }}^{\mathrm{rec}_{1}}(\boldsymbol{\theta})$.

Finally, by noting that for coherent rectilinear sources, $\widetilde{\mathbf{H}}=\kappa_{c} \mathbf{c c}^{T}$ in (11) gives $\mathrm{CRB}_{\text {sto }}^{\mathrm{rec}_{2}}(\boldsymbol{\omega})=\mathrm{CRB}_{\mathrm{sto}}^{\mathrm{rec}_{1}}(\boldsymbol{\omega})$, It follows then that $\mathrm{CRB}_{\text {sto }}^{\mathrm{rec}_{2}}(\boldsymbol{\theta})=\mathrm{CRB}_{\text {sto }}^{\mathrm{rec}_{1}}(\boldsymbol{\theta})$.

\section{CONCLUSION}

Closed-form expressions of the SCRB of DOA estimation for rectilinear sources have been directly derived form the extended Slepian-Bangs formula, including the case of prior knowledge of uncorrelated or coherent rectilinear sources. Analytical and numerical comparisons with the DCRB and the SCRB for noncircular sources have shown in particular that the DCRB may be very optimistic. 


\section{REFERENCES}

[1] P. Gounon, C. Adnet, and J. Galy, "Localization angulaire de signaux non circulaires," Traitement du Signal, vol. 15, no. 1, pp. 17-23, 1998.

[2] H. Abeida and J.P. Delmas, "MUSIC-like estimation of direction of arrival for non-circular sources," IEEE Trans. Signal Process., vol. 54, no. 7, pp. 2678-2690, July 2006.

[3] P. Chargé, Y. Wang, and J. Saillard, "A non-circular sources direction finding method using polynomial rooting," Signal Process., vol. 81, pp. 1765-1770, 2001.

[4] A. Zoubir, P. Chargé, and Y. Wang, "Non circular sources localization with ESPRIT," in Proc. Eur. Conf. Wireless Technology (ECWT), Munich, Germany, Oct. 2003.

[5] M. Haardt and F. Roemer, "Enhancements of unitary esprit for noncircular sources," Proc. ICASSP, vol. 2,pp. 101-104, Montreal, QC, Canada, May 2004.

[6] J. Steinwandt, F. Roemer, M. Haardt, and G. Del Galdo, "Rdimensional ESPRIT-type algorithms for strictly second-order noncircular sources and their performance analysis," IEEE Trans. Signal Processing, vol. 62, no. 18, pp. 4824-4838, Sept. 2014.

[7] J.P. Delmas and H. Abeida, "Stochastic Cramer-Rao bound for noncircular signals with application to DOA estimation," IEEE Trans. Signal Process., vol. 52, no. 11, pp. 3192-3199, Nov. 2004.

[8] H. Abeida and J.P. Delmas, "Efficiency of subspace-based DOA estimators," Signal Processing, vol. 87, no. 9, pp. 2075-2084, Sept. 2007.

[9] H. Abeida and J.P. Delmas, "Bornes de Cramer Rao déterministe et stochastique de DOA de signaux rectilignes non corrélés," Proc. GRETSI, Troyes, Sept. 2007,

[10] F. Roemer and M. Haardt, "Deterministic Cramer Rao bounds for strict sense noncircular sources," Proc. Internat. ITG/IEEE Worshop on Smart Antennas (WSA'07), Vienne, Austria, Feb. 2007.

[11] J. Steinwandt, F. Roemer, M. Haardt, and G. Del Galdo, "Deterministic Cramer-Rao bound for strictly non-circular sources and analytical analysis of the achievable gains," IEEE Trans. Signal Process., vol. 64, no. 17, pp. 4417-4431, Sept. 2016.

[12] D.T. Vu, A. Renaux, R. Boyer, and S. Marcos "Some results on the Weiss-Weinstein bound for conditional and unconditional signal models in array processing," Signal Processing, vol. 95, pp. 126-148, Feb. 2014.

[13] P. Stoica and A. Nehorai, "Performance study of conditional and unconditional direction of arrival estimation," IEEE Trans. Signal Process., vol. 38, no. 10, pp. 1783-1795, Oct. 1990.

[14] D. Slepian, "Estimation of signal parameters in the presence of noise," in Trans. IRE Prof. Grop Inform. Theory PG IT-3, pp. 68-89, 1954.

[15] W.J. Bangs "Array processing with generalized beamformers," Ph.D. thesis Yale University, New Haven, CT, 1971.

[16] P. Stoica, A.G. Larsson, and A.B. Gershman, "The stochastic CRB for array processing: a textbook derivation," IEEE Signal Process. letters, vol. 8, no. 5, pp. 148-150, May 2001

[17] M. Jansson, B. Göransson, and B. Ottersten, "Subspace method for direction of arrival estimation of uncorrelated emitter signals," IEEE Trans. Signal Process., vol. 47, no. 4, pp. 945-956, April 1999.

[18] J. Sheinvald, M. Wax, and A.J. Weiss, "On maximum-likelihood localization of coherent signals," IEEE Transactions on Signal Processing, vol. 44, no. 10, pp. 2475-2482, Oct. 1996.

[19] J. R. Schott, Matrix analysis for statistics, Wiley, New York, 1997.

[20] P. Stoica and R. Moses, Spectral analysis of signals, Prentice-Hall, Upper Saddle River, NJ, 2005.

[21] P. Stoica and A. Nehorai, "MUSIC, maximum likelihood, and Cramer-Rao bound: Further results and comparisons," IEEE Trans. Signal Process., vol. 38, no. 12, pp. 2140-2150, Dec. 1990.

[22] R.A. Horn and C.R. Johnson, Matrix analysis, Cambridge University Press, 1996.

[23] H. Abeida and J.P. Delmas, Detailed proofs of Theorems 1 and 2 are available at http://www-public.int-evry.fr/ delmas/ 